\title{
Distributed load sensor by use of a chirped moiré fiber Bragg grating
}

\author{
Andrew Michael Gillooly, Helen Dobb, Lin Zhang, and lan Bennion
}

\begin{abstract}
A chirped moiré fiber Bragg grating has been demonstrated to be capable of measuring the magnitude, position, and footprint of a transverse load. The device provides an average spatial resolution of $164 \mu \mathrm{m}$ and has a load accuracy of $0.15 \mathrm{~N} / \mathrm{mm}$, or $50 \mu \varepsilon$. (C) 2004 Optical Society of America

OCIS codes: $\quad 050.1590,050.2770,050.5080,060.2310,060.2370,120.4120$.
\end{abstract}

\section{Introduction}

There are a variety of ways in which chirped moiré fiber Bragg gratings (CMFBGs) can be fabricated. ${ }^{1-3}$ Such gratings have been used as wide stop-band transmission filters, ${ }^{2}$ wavelength-division multiplexing channel selectors, ${ }^{4}$ distributed strain sensors, ${ }^{5}$ and distributed temperature sensors. ${ }^{6}$ We are aware of no research conducted on the use of CMFBGs as distributed load sensors. Load sensors have been studied by use of a number of fiber grating structures, including uniform fiber Bragg gratings, ${ }^{7}$ piphase shifted fiber Bragg gratings, ${ }^{8}$ chirped fiber gratings, ${ }^{9}$ sampled fiber-Bragg gratings, ${ }^{10}$ and longperiod gratings. ${ }^{11,12}$ Most of these techniques allow either the applied load or the position of the load to be measured but never both simultaneously. The method proposed theoretically by Torres and Valente $^{7}$ and demonstrated experimentally by Tjin et al. ${ }^{9}$ does allow both load and position to be measured, but with this method it proves difficult to define the footprint accurately. In this paper we present a distributed load sensor that is capable of accurately retrieving the magnitude, position, and footprint of a load that is being applied transversely to a fiber.

\section{Moire Grating}

A CMFBG was fabricated with a 244-nm frequencydoubled cw Coherent FreD laser by a phase-mask fabrication technique. The 25-mm-long grating was

The authors are with the Photonics Research Group, Aston University, Aston Triangle, Birmingham B4 7ET, United Kingdom. A. M. Gillooly's e-mail address is gillooam@aston.ac.uk.

Received 11 June 2004; accepted 13 August 2004.

0003-6935/04/356454-04\$15.00/0

(C) 2004 Optical Society of America inscribed into hydrogenated SMF-28 single-mode fiber by a double scan technique. ${ }^{3}$ An initial chirped grating was written, the fiber was strained, and then a second chirped grating was written directly over the first grating. The straining gave a 5 -nm difference in the central wavelength of the two gratings. As the second grating was written with a period that was different from that of the first grating, a beat effect was created by the superposition of the two gratings' fringe patterns. Hence phase shifts were introduced at the zero points of the beat effect that acted as passbands, thus creating the moiré structure (Fig. 1). The subsequent refractive-index profile is defined by ${ }^{13}$

$$
\Delta n(z)=2 \delta n\left[1+\cos \left(\frac{2 \pi z}{\Lambda_{a}}\right) \cos \left(\frac{2 \pi z}{\Lambda_{b}}\right)\right],
$$

where $\delta n$ is the dc offset of the refractive index, $z$ is the position along the fiber axis, and $\Lambda_{a}$ and $\Lambda_{b}$ are defined by Eqs. (2) and (3), respectively,

$$
\begin{aligned}
& \Lambda_{a}=\frac{2 \Lambda_{1} \Lambda_{2}}{\left(\Lambda_{1}+\Lambda_{2}\right)}, \\
& \Lambda_{b}=\frac{2 \Lambda_{1} \Lambda_{2}}{\left(\Lambda_{1}-\Lambda_{2}\right)},
\end{aligned}
$$

where $\Lambda_{1}$ and $\Lambda_{2}$ are the periods of the two superimposed gratings. For a chirped grating they are expressed as a function of $z . \quad \Lambda_{b}$ is the period of the slowly varying envelope. From this the spatial separation of each passband of the device is given by

$$
\Delta z=\Lambda_{b} / 2
$$




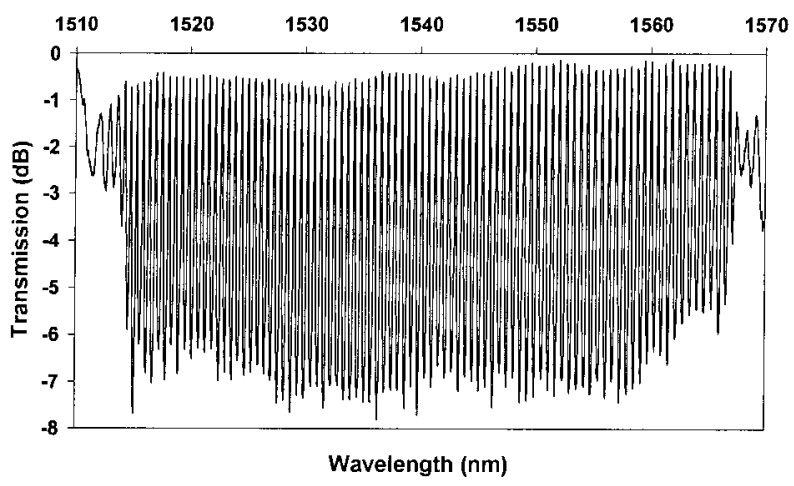

Fig. 1. Transmission spectrum of a CMFBG fabricated by the double-scan technique.

It is then possible to calculate the number of passbands formed in the CMFBG from

$$
N=L / \Delta z \text {. }
$$

Because the grating is chirped, the spacing between the passbands, $\Delta z$, varies slightly with position. For the CMFBG shown in Fig. 1, the spacing at $1515 \mathrm{~nm}$ is $158 \mu \mathrm{m}$, and at $1565 \mathrm{~nm}$ the spacing is $169 \mu \mathrm{m}$, as calculated from Eq. (4). This implies that the spatial resolution of the device is different for each point along the grating length. Practically, this is not a problem because the difference is insignificant to the size of any loading element. The mean spatial resolution of the device is $164 \mu \mathrm{m}$. This is the highest spatially resolved moiré grating of which we are aware.

\section{Position and Footprint Sensing}

A loading device was used to apply a load transverse to the optical fiber's longitudinal axis. The device consisted of two parallel metallic plates with the CMFBG between them. To maintain an even load distribution on the grating, a second piece of fiber was placed alongside, as shown in Fig. 2. This ensured that any load was applied through the vertical plane with no horizontal component. The CMFBG was connected to an Agilent 8164A light-wave measure-

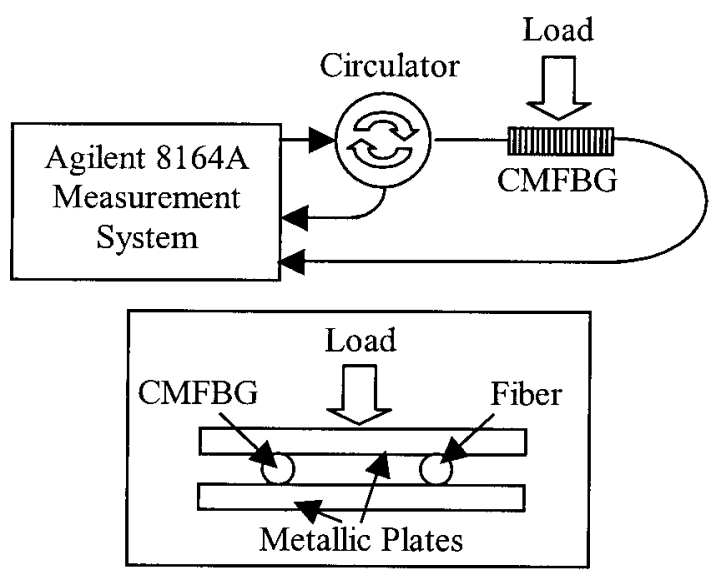

Fig. 2. CMFBG loading and interrogation setup.

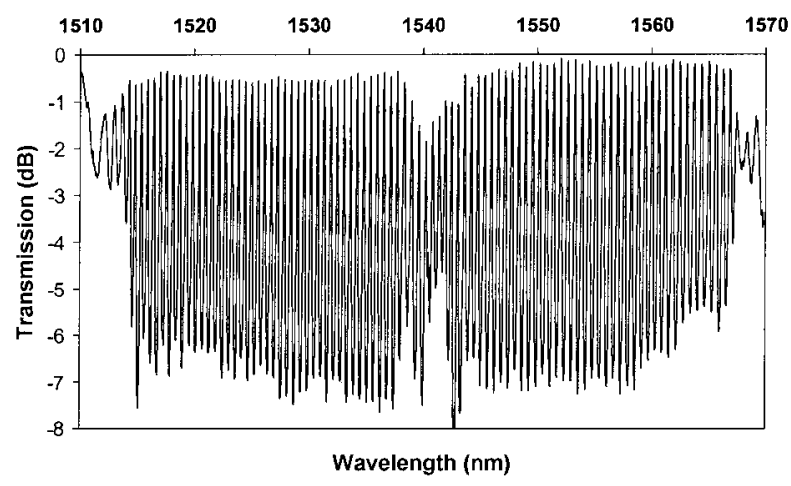

(a)

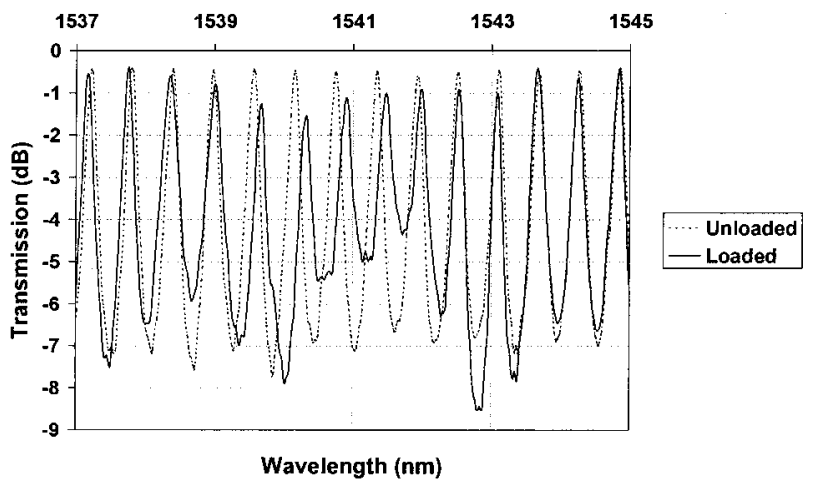

(b)

Fig. 3. (a) Transmission spectrum of a CMFBG with a loading element placed in the middle of the grating. (b) Magnification of the central region of the sensor.

ment system and a circulator for transmission and reflection measurements.

When a load was applied to the CMFBG a redshift in wavelength occurred near the point where the load was applied, as shown in Refs. 7 and 9 and demonstrated in Fig. 3. An important point to note is that this technique of measuring the wavelength shift is not capable of detecting the load magnitude accurately. In Section 4 below, we describe an accurate way to sense the load.

Because the grating is chirped, each wavelength corresponds to a physical position along the grating. As the phase shifts occur at discrete wavelengths within the fiber, their physical position may be calculated. The wavelength of the grating can be converted to position along the grating by use of the equation $^{14}$

$$
x=\frac{\lambda_{0}-\lambda_{x}}{n_{\text {eff }} C},
$$

where $\lambda_{0}$ is the starting wavelength of the grating, $\lambda_{x}$ is the wavelength at any position along the grating, $n_{\text {eff }}$ is the effective refractive index of the grating, and $C$ is the chirp rate. By measuring the position of the fringes for which this redshift occurs, one may find the position and the footprint of the load on the grating (Fig. 4). 


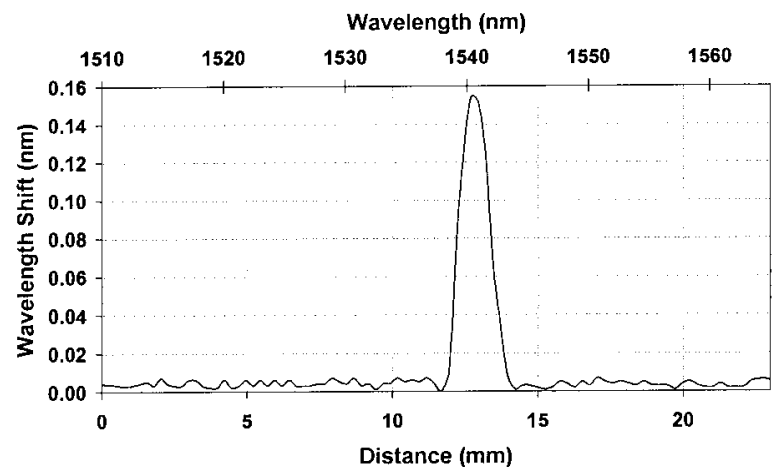

Fig. 4. Magnitude of the redshift that is due to the loading element, showing the footprint and the position of the load.

Using Eq. (6), we calculated the footprint of the loading element as $2.54 \pm 0.16 \mathrm{~mm}$, in excellent agreement with the actual measurement of $2.50 \mathrm{~mm}$.

\section{Load Sensing}

The magnitude of the load may be calculated by use of the effect of birefringence as demonstrated by LeBlanc et al. 8 As load is applied to the fiber, birefringence occurs owing to the nonisotropic nature of the load. This causes the phase shifts to be split into two peaks, which correspond to the induced fast and slow axes of the grating as shown in Fig. 5.

Figure 5 shows not only the peak splitting but also the red wavelength shift and a reduction in the level of light transmitted. This is due partly to the deformation of the core's cross section and partly to the displacement of the core in the plane to which the load is applied, both of which will decrease the guiding ability of the fiber, therefore increasing the optical transmission loss of the fiber over the area where the load is applied. The birefringence, $B$, that causes the peak splitting can be calculated from the equation 8

$$
B=\frac{\Delta n}{\bar{n}}=\frac{\Delta \lambda}{\bar{\lambda}},
$$

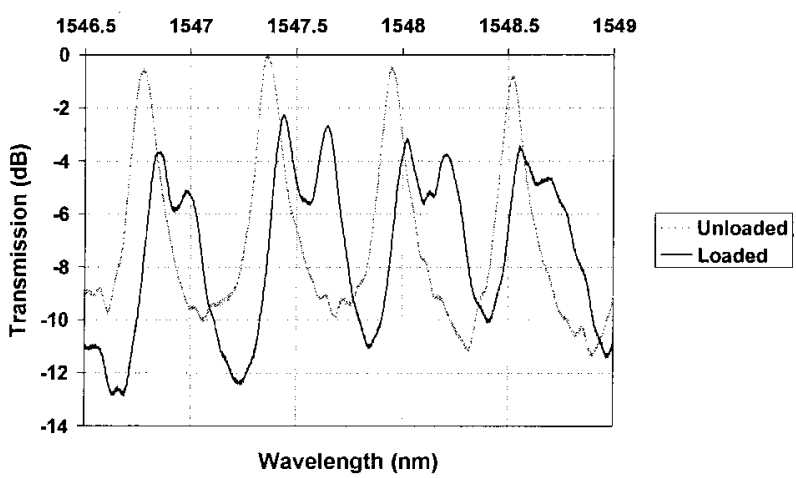

Fig. 5. Comparison of phase shifts before and after a load is applied, showing the peak splitting that is due to the induced birefringence.

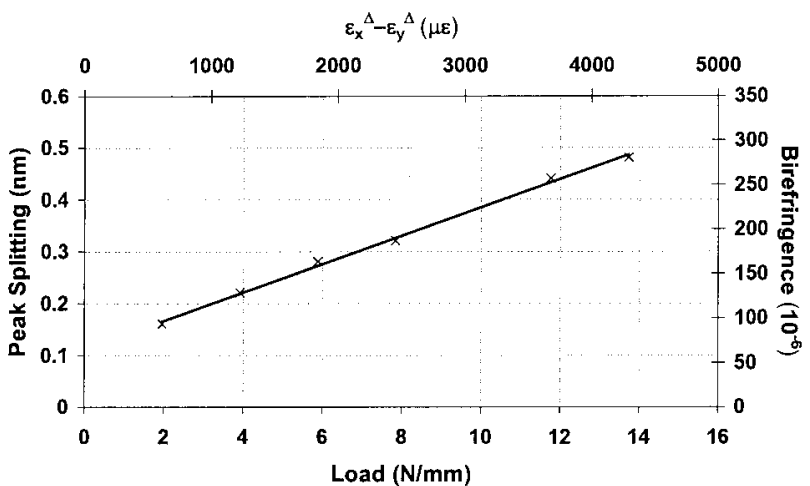

Fig. 6. Linear dependence of birefringence on the applied load.

where

$$
\Delta n=n_{1}-n_{2}
$$

is the difference in the index of refraction between the slow and fast axes of the grating, $\bar{n}$ is the average index of refraction of the grating core, and

$$
\bar{\lambda}=\frac{\lambda_{1}+\lambda_{2}}{2}
$$

is the average wavelength of the grating, where $\lambda_{1}$ and $\lambda_{2}$ are the wavelengths of the two split peaks. It is useful to convert the applied load to a transverse strain measurement by using the following equation $^{15}$ :

$$
\left(\varepsilon_{x}{ }^{\Delta}-\varepsilon_{y}{ }^{\Delta}\right)=\frac{8\left(1+v_{f}\right)}{E_{f}} \frac{F}{\pi d_{f}},
$$

where $v_{f}=0.17$ is the Poisson ratio of the optical fiber, $F$ is the force per unit length, $E_{f}=70.3 \mathrm{GPa}$ is the Young modulus of the fiber, and $d_{f}=125 \mu \mathrm{m}$ is the diameter of the fiber. The strain measurement gives a physical measurement of the conditions within the fiber; a load measurement would give a measurement of the conditions on the surface of the fiber. The linear dependence of the birefringence induced on the load, shown in Fig. 6, allows this technique to be readily applicable to a real-life device.

The Agilent measurement system is capable of

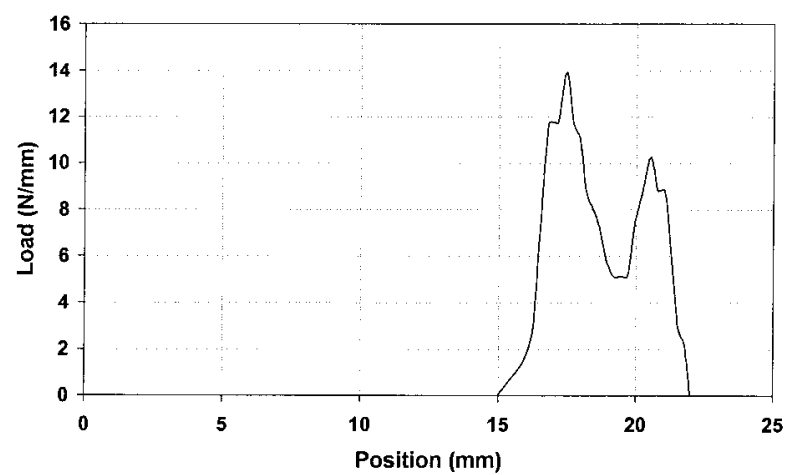

Fig. 7. Distributed load of a 7-mm-wide loading element. 
measuring the peak separation to a resolution of 0.2 $\mathrm{pm}$. As the gradient of the slope is $27.3 \mathrm{pm} /(\mathrm{N} /$ $\mathrm{mm}$ ), the accuracy of the loading experiment is 0.15 $\mathrm{N} / \mathrm{mm}$, thus giving a transverse strain accuracy of 50 $\mu \varepsilon$. This then allows a distributed measurement of the load of an object on top of the CMFBG to be calculated, as shown in Fig. 7.

The loading element was a 7-mm-wide piece of aluminum placed at one end of the CMFBG. Figure 7 indicates that the maximum load was $14 \mathrm{~N} / \mathrm{mm}$ and that there was not a constant load across the entire length of the aluminum block; this is probably so because the surface of the block was not perfectly flat.

\section{Conclusions}

A device to give a distributed measurement of the magnitude, position, and footprint of a transverse load has been experimentally demonstrated. The CMFBG presented has an extremely high average spatial resolution of $164 \mu \mathrm{m}$ and a loading accuracy of $0.15 \mathrm{~N} / \mathrm{mm}$, or $50 \mu \varepsilon$. The device covers a short spatial range, but it is possible to fabricate such gratings over a greater length. Such a grating would lose spatial resolution if it were fabricated with the same chirp rate. Therefore there is a direct trade-off between the spatial resolution and the length of the grating. However, any application that required a longer grating would probably not require such high resolution.

\section{References}

1. S. Legoubin, E. Fertein, M. Douay, P. Bernage, P. Niay, F. Bayon, and T. Georges, "Formation of moiré grating in core of germanosilicate fibre by transverse holographic double exposure method," Electron. Lett. 27, 1945-1946 (1991).

2. L. Zhang, K. Sugden, I. Bennion, and A. Molony, "Widestopband chirped fibre moiré grating transmission filters," Electron. Lett. 31, 477-479 (1995).

3. L. A. Everall, K. Sugden, J. A. R. Williams, and I. Bennion, "Fabrication of multipassband moire resonators in fibres by the dual-phase-mask exposure method," Opt. Lett. 22, 14731475 (1997).
4. A. Carballar, M. A. Muriel, and J. Azaña, "WDM channel selector based on transmissive chirped moiré fibre grating," Electron. Lett. 35, 386-388 (1999).

5. K. E. Chisholm, K. Sugden, I. J. Murgatroyd, B. Sundström, B. Lindström, and L. Zhang, "Application of chirped moiré fibre Bragg gratings to quasi-distributed monitoring of strains experienced by fibres during processing," presented at the 14th International Conference on Optical Fiber Sensors (OFS), Venice, Italy, 11-13 October 2000.

6. K. E. Chisholm, K. Sugden, L. Zhang, and I. Bennion, "Novel high spatial resolution and temperature sensor using chirped moiré fibre gratings," paper presented at the OSA Topical Meeting on Bragg Gratings, Photosensitivity, and Poling in Glass Waveguides (BGPP), Stuart, Fla., 23-25 September 1999.

7. P. Torres and L. G. C. Valente, "Spectral response of locally pressed fiber Bragg grating," Opt. Commun. 208, 285-291 (2002).

8. M. LeBlanc, S. T. Vohra, T. E. Tsai, and E. J. Friebele, “Transverse load sensing by use of pi-phase-shifted fiber Bragg gratings," Opt. Lett. 24, 1091-1093 (1999).

9. S. C. Tjin, L. Mohanty, and N. Q. Ngo, "Pressure sensing with embedded chirped fiber grating," Opt. Commun. 216, 115-118 (2003).

10. X. W. Shu, K. Chisholm, I. Felmeri, K. Sugden, A. Gillooly, L. Zhang, and I. Bennion, "Highly sensitive transverse load sensing with reversible sampled fiber Bragg gratings," Appl. Phys. Lett. 83, 3003-3005 (2003).

11. Y. Liu, L. Zhang, and I. Bennion, "Fibre optic load sensors with high transverse strain sensitivity based on long-period gratings in B/Ge co-doped fibre," Electron. Lett. 35, 661-663 (1999).

12. L. Zhang, "Design and realisation of long-period grating devices in conventional and high birefringence fibers and their novel applications as fibre optic load sensors," IEEE J. Sel. Top. Quantum Electron. 5, 1371-1378 (1999).

13. A. Othonos and K. Kalli, Fiber Bragg Gratings (Artech House, Boston, Mass., 1999).

14. A. M. Gillooly, K. E. Chisholm, L. Zhang, and I. Bennion, "Chirped fibre Bragg grating optical wear sensor," Meas. Sci. Technol. 15, 885-888 (2004).

15. R. B. Wagreich, W. A. Atia, H. Singh, and J. S. Sirkis, "Effects of diametric load on fibre Bragg gratings fabricated in low birefringent fibre," Electron. Lett. 32, 1223-1224 (1996). 